\title{
Conjugation of DNA to Silanized Colloidal Semiconductor Nanocrystalline Quantum Dots
}

\author{
Wolfgang J . Parak, ${ }^{\dagger, \neq, \S}$ Daniele Gerion, ${ }^{\dagger, t, \S}$ Daniela Zanchet,, Anke S. Woerz,, \\ Teresa Pellegrino, $\$$ Christine Micheel,,,$\S$ Shara C. Williams, ${ }^{\ddagger}, \S$ Markus Seitz, \\ Richard E. Bruehl, $\neq, \S$ Zev Bryant," Carlos Bustamante," \\ Carolyn R. Bertozzi,,,$\S$, and A. Paul Alivisatos*, $*, \S$ \\ Materials Science Division, Lawrence Berkel ey National Laboratory, Berkeley, California, \\ Department of Chemistry, University of California, Berkeley, California, Institut fuer \\ Angewandte Physik, Ludwig-Maximilians-Universität, München, Germany, and Department of \\ Molecular and Cell Biology, University of California, Berkeley, California
}

Received September 12, 2001. Revised Manuscript Received February 22, 2002

\begin{abstract}
Water-soluble, highly fluorescent, silanized semiconductor nanocrystals with different surface charges were synthesized. To covalently attach the nanocrystals to biological macromolecules with a variety of mild coupling chemistries, the outermost siloxane shells were derivatized with thiol, amino, or carboxyl functional groups. Single- or double-stranded DNA was coupled to the nanocrystal surfaces by using commercially available bifunctional cross-linker. Conjugation had little effect on the optical properties of the nanocrystals, and the resulting conjugates were more stable than previously reported systems. By using the strategies developed in this study, most biomolecules can be covalently coupled to semiconductor nanocrystals. These nanocrystal-DNA conjugates promise to be a versatile tool for fluorescence imaging and probing of biological systems.
\end{abstract}

\section{Introduction}

Nanocrystals derivatized with biological molecules have been applied in materials science and biological research in recent years. ${ }^{1}$ For example, ordered patterns of metallic nanocrystals have been constructed by using oligonucleotides as molecular scaffolds to direct the assembly process, ${ }^{2-4}$ and biotinylated, oligonucleotidedecorated, or antibody-bearing semiconductor nanocrystals spanning a spectrum of colors have been used as fluorescent probes for cellular imaging. ${ }^{5-7}$ A key requirement for the successful use of nanocrystals in both of these applications was aqueous solubility. Methods for conferring water solubility on colloidal semiconductor quantum dots prepared in hydrophobic environments remains an active topic of research. Thus, to confer water solubility, the widely used hydrophobic CdSe/ZnS (core/shell) nanocrystals were coated with an

* Corresponding author.

† Both authors contributed equally to this work.

₹ Lawrence Berkeley National Laboratory.

$\S$ Department of Chemistry, University of California, Berkeley.

" Ludwig-Maximilians-Universität.

" Department of Molecular and Cell Biology, University of California, Berkeley.

(1) Alivisatos, P. Pure Appl. Chem. 2000, 72, 3-9.

(2) Mirkin, C. A.; Letsinger, R. L.; Mucic, R. C.; Storhoff, J . J . Nature 1996, 382, 607-609.

(3) Alivisatos, A. P.; J ohnsson, K. P.; Peng, X.; Wilson, T. E.; Loweth,

C. J .; Bruchez, M. P., J r.; Schultz, P. G. Nature 1996, 382, 609-611.

(4) Loweth, C. J .; Caldwell, W. B.; Peng, X. G.; Alivisatos, A. P.; Schultz, P. G. Angew. Chem. Int. Ed. Engl. 1999, 38, 1808-1812.

(5) Chan, W. C. W.; Nie, S. Science 1998, 281, 2016-2018.

(6) Bruchez, M. J .; Moronne, M.; Gin, P.; Weiss, S.; Alivisatos, A. P. Science 1998, 281, 2013-2016.

(7) Pathak, S.; Choi, S.-K.; Arnheim, N.; Thompson, M. E. J . Am. Chem. Soc. 2001, 123, 4103-4104. additional outer hydrophilic shell.,5,6,8-15 Biological molecules such as biotin or oligonucleotides have been coupled to the surface of water-soluble semiconductor nanocrystals by using mono- or bifunctional cross-linker molecules, ${ }^{5-7}$ or have been adsor bed by el ectrostatic and hydrophobic attraction. ${ }^{10,11}$

Surface silanization is a powerful strategy for preparing water-soluble nanocrystals that offers several advantages. ${ }^{6,12-15} \mathrm{First}$, the siloxane shell is extensively cross-linked, so that the stability of the system does not depend on only one or a few bonds. Second, silanized nanocrystals remain stable in a range of biological environments including physiological and supraphysiological salt concentrations ( $\gg 100 \mathrm{mM}$ ). ${ }^{15}$ Finally, the surface chemistry involved in the bioconjugation of glass surfaces is well established and can be readily extended to the silanized nanocrystals described here. Disadvantages of surface silanization include the possibilities of

(8) Mitchell, G. P.; Mirkin, C. A.; Letsinger, R. L. J . Am. Chem. Soc. 1999, 121, 8122-8123.

(9) Chen, C.-C.; Yet, C.-P.; Wang, H.-N.; Chao, C.-Y. Langmuir 1999, 15, 6845-6850.

(10) Mattoussi, H.; Mauro, J . M.; Goldman, E. R.; Anderson, G. P.; Sundar, V. C.; Mikulec, F. V.; Bawendi, M. G. J . Am. Chem. Soc. 2000, $122,12142-12150$.

(11) Mattoussi, H.; Mauro, J . M.; Goldman, E. R.; Green, T. M.; Anderson, G. P.; Sundar, V. C.; Bawendi, M. G. Phys. Status Śolidi B 2001, 224, 277-283.

(12) Correa-Duarte, M. A.; Giersig, M.; Liz-Marzán, L. M. Chem Phys. Lett. 1998, 286, 497-501.

(13) Mulvaney, P.; Liz-Marzán, L. M.; Giersig, M.; Ung, T. J . Mater. Chem. 2000, 10, 1259-1270.

(14) Rogach, A. L.; Nagesha, D.; Ostrander, J. W.; Giersig, M.; Kotov, N. A. Chem. Mater. 2000, 12, 2676-2685.

(15) Gerion, D.; Pinaud, F.; Williams, S. C.; Parak, W. J .; Zanchet, D.; Weiss, S.; Alivisatos, A. P. J . Phys. Chem. B 2001,105, 8861-8871. 


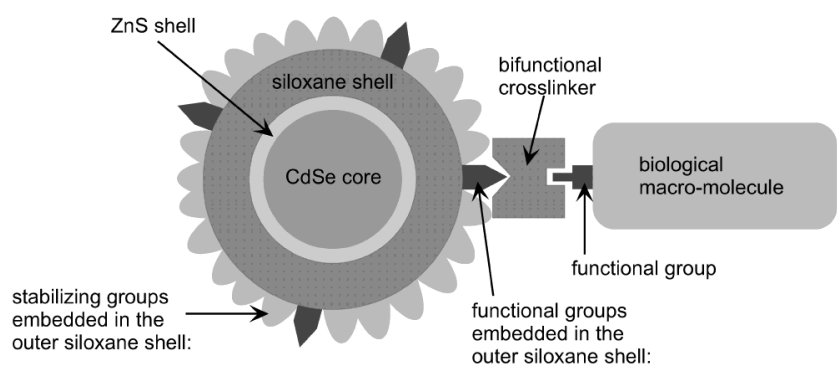

Figure 1. Scheme of a biomolecule-nanocrystal conjugate. $\mathrm{CdSe/ZnS}$ (core/shell) nanocrystals are embedded in a siloxane shell, and phosphonate, PEG, or ammonium groups on the outer siloxane surface act as stabilizing groups to maintain water solubility. Thiols, amines, or carboxyl groups are incorporated in the siloxane shell as functional groups.

nanocrystal aggregation caused by interparticle crosslinking, and the introduction of additional heterogeneity in nanocrystal size and surface-charge distribution. These challenges of nanocrystal synthesis, however, can be controlled by ensuring a sufficiently dilute reaction and have minimal effect on the successful use of watersoluble, silanized nanocrystals in general biological imaging and probing applications.

The study reported here has two aims. The first involves describing a generalized method for the synthesis of silanized, water-soluble CdSe/ZnS nanocrystals with different surface charges and chemical functionalities. Although negatively charged nanocrystals have been described previously, ${ }^{15}$ this study reports for the first time the production of positively charged and almost neutral nanocrystals. The ability to tune the surface charge of the nanocrystals and incorporate any of the most relevant biological functional groups $\left(-\mathrm{COOH},-\mathrm{NH}_{2},-\mathrm{SH}\right)$ allows for the generation of tailored siloxane shells with highly biocompatible surfaces. The second aim of this study is to develop a protocol for covalently conjugating DNA to the nanocrystal surface (Figure 1). Covalent coupling makes nanocrystal-DNA conjugates highly stable in el ectrolytic solution. The present experiments potentially enable conjugation to a wide range of biomolecules, including those that are components of receptor-ligand pairs such as streptavidin-biotin and antibody-antigen, in addition to the ol igonucleotide-complementary oligonucleoti de case that is demonstrated here. Because nanocrystals have different photophysical properties than organic dyes, including greater resistance to photobleaching, these conjugation experiments further devel op this tool kit for fluorescence detection in biology.

\section{Experimental Section}

Nanocrystal Silanization. The details of growing a negatively charged siloxane shell around CdSe/ZnS (core/shell) nanocrystals have been described recently. ${ }^{15}$ In brief, in the first step, hydrophobic CdSe/ZnS nanocrystals are primed with mercaptosilane (mercaptopropyltrimethoxysilane, no. 175617, Sigma-Aldrich, Milwaukee, WI) in an alkaline methanol solution. Under these conditions, the mercapto groups bind to the ZnS surface and displace the original surfactant molecules (trioctyl phosphineoxide, TOPO, no. 346187, SigmaAldrich). Heating the reaction to $60^{\circ} \mathrm{C}$ promotes cross-linking of the silanol groups and formation of a lattice of siloxane bonds encasing the nanocrystal quantum dots. In the second step, negatively charged phosphonate silane (trihydroxysilylpropyl methylphosphonate monosodium salt, no. 435716,
Sigma-Aldrich) and mercaptosilane are added and cross-linked to stabilize the nanocrystals in aqueous solution and to introduce surface thiols as functional groups, respectively. In the final step, unreacted silanol groups were quenched by the addition of chlorotrimethylsilane (C7,285-4, Sigma-Aldrich) to afford a stable outer shell.

To obtain positively charged or virtually neutral nanocrystals, the phosphonate silane used in the second step of the silanization was replaced with either poly(ethylene glycol) (PEG)-silane (methoxypolyethyleneoxy propyltrimethoxysilane, no. SI M6492.7, Gelest, Tullytown, PA) or a 1:1 mixture of PEG-silane and ammoniumsilane (trimethoxysilylpropyltrimethylammonium chloride, no. SIT8415.0, Gelest). ${ }^{16}$ Because mercaptosilane is used for the first step of the silanization, homogeneous thiol-bearing nanocrystals can be obtained easily. However, if in the second step aminosilane is added, excess mercaptosilane from the first step will result in a mixture of surface amines and thiols. To obtain a homogeneous amine shell, residual thiol groups are converted to amines by reacting the nanocrystals with iodoethyltrifluoroacetamine (no. 23010, Pierce, Rockford, IL) in a postsynthetic manipulation according to the manufacturer's protocol. In a similar manner, nanocrystals with a homogeneous carboxyl surface can be generated by converting the surface thiols to carboxyl groups by using maleimidopropionic acid (no. 394815, Sigma-Aldrich or no. 22296, Pierce) according to the manufacturer's protocol.

Two procedures have been used for the removal of excess silica. One involves purification of the nanocrystals on an agarose gel, which eliminates most of the small silica nanocrystals and nanocrystal aggl omerates. ${ }^{15} \mathrm{An}$ alternative method uses size exclusion chromatography (Sephadex G-200 filled columns, no. 84958, Fluka, Milwaukee, WI), which also removes small silica nanocrystals and nanocrystal agglomerates. To verify removal of excess silane, the number of thiol molecules per nanocrystal in the solution was determined by using Ellman's reagent (dithiobisnitrobenzoic acid, no. D8130, Sigma-Aldrich or no. 22582, Pierce) according to the manufacturer's protocol. 17,18

Bioconjugation. Thiol-bearing nanocrystals stabilized with PEG or phosphonate were derivatized with amino-modified oligonucleotides in a two-step procedure using the heterobifunctional cross-linker sulfo-SMCC (maleimidomethylcyclohexanecarboxylic acid sulfohydroxysuccinimide ester sodium salt, no. M6035, Sigma-Aldrich). Sulfo-SMCC has an Nhydroxysuccinimide (NHS) ester at one end, which reacts with a primary amine to form an amide bond and a maleimide group at the other, which reacts with a thiol to form a thioether. In the first step, the mal eimide functional group is introduced into the amino-modified oligonucleotide by con-

(16) All experimental steps have been described in detail in a recent publication for phosphonate-stabilized nanocrystals. ${ }^{15} \mathrm{CdSe} / \mathrm{ZnS}$ nanocrystals $(3-5 \mathrm{~mL}$ ) dissolved in butanol/trioctylphosphine oxide were precipitated with anhydrous methanol. The wet precipitate was dissolved in $320 \mu \mathrm{l}$ of mercaptosilane. After vortexing, $16 \mu \mathrm{l}$ of $25 \%$ tetramethylammonium hydroxide in methanol was added and the solution became optically clear. This mixture was diluted with $400 \mathrm{~mL}$ of anhydrous methanol basified with $2.4 \mathrm{~mL}$ of $25 \%$ tetramethylammonium hydroxide. After 30-60 min of stirring, the solution was heated to $65{ }^{\circ} \mathrm{C}$ for $30 \mathrm{~min}$. After cooling to room temperature, 360 $\mathrm{mL}$ of methanol, $40 \mathrm{~mL}$ of Millipore water, $2.4 \mathrm{~mL}$ of phosphonate silane, and $80 \mu \mathrm{l}$ of mercaptosilane were added, stirred for ca. $2 \mathrm{~h}$, heated to $65^{\circ} \mathrm{C}$ for about $5 \mathrm{~min}$, and cooled to room temperature. The remaining silanol groups were quenched with a mixture $64 \mathrm{~mL}$ of methanol, and $6.4 \mathrm{~mL}$ of chlorotrimethylsilane that had been neutralized with tetramethylammonium hydroxide pentahydrate, and then stirred again for ca. $2 \mathrm{~h}$. The solution was heated to ca. $65^{\circ} \mathrm{C}$ for ca. $30 \mathrm{~min}$, and then left at room temperature for $2-4$ days under stirring The final concentrations of the silane reagents and nanocrystals in the alkaline methanol mixture were: CdSe/ZnS nanocrystals, 20-200 $\mathrm{nM}$; mercaptosilane, $1.9 \mathrm{mM}$ (first step), $0.5 \mathrm{mM}$ (second step) phoshonate silane, $14 \mathrm{mM}$; chlorotrimethylsilane, $56 \mathrm{mM}$. For virtually neutral nanocrystals PEG-silane instead of phosphonate silane with the same concentration (14 mM) was used. For positively charged nanocrystals ammonium silane (7 mM) and PE G-silane (7 mM) instead of phosphonate silane was used.

(17) Riddles, P. W.; Blakeley, R. L.; Zerner, B. Anal. Biochem. 1979, 94, 75-81.

(18) Ellman, G. L. Arch. Biochem. Biophys. 1959, 82, 70-77. 


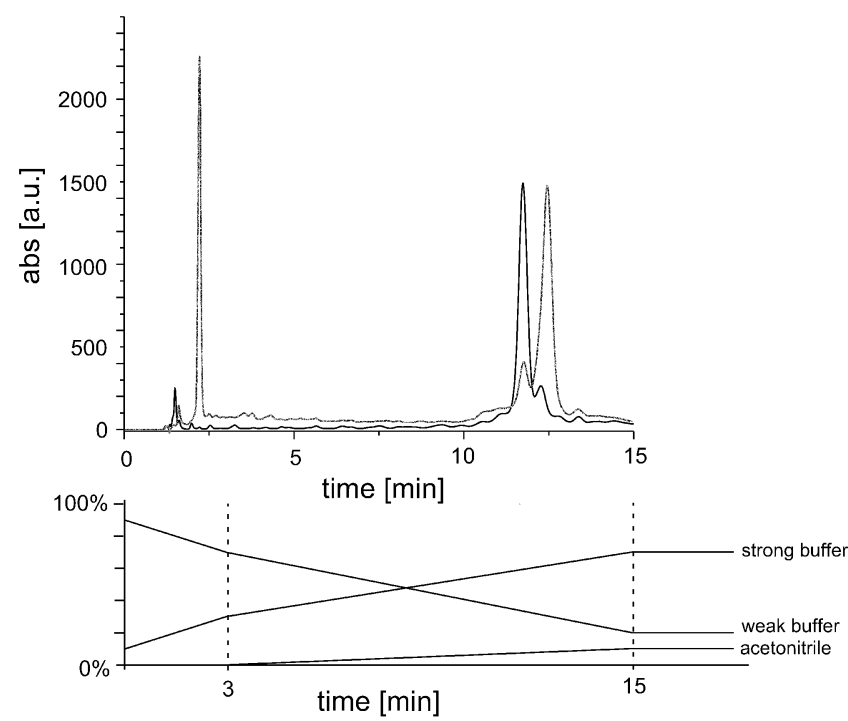

Figure 2. HPLC purification of sulfo-SMCC-activated DNA. To separate excess sulfo-SM CC from DNA a gradient of strong buffer (phosphate buffer, $1 \mathrm{M} \mathrm{NaCl}$ ), weak buffer (phosphate buffer, no $\mathrm{NaCl}$ ), and acetonitrile was applied. Free DNA (21 bases) elutes at about $11.5 \mathrm{~min}$. The elution profile of sulfoSMCC-activated DNA shows three peaks. After ca. 2 min free sulfo-SMCC is eluted. After ca. $11.5 \mathrm{~min}$, unreacted DNA (small peak), and after ca. $12.5 \mathrm{~min}$, sulfo-SMCC-activated DNA (big peak) is eluted. With the protocol described here, up to $80 \%$ of the DNA could be activated. Only the activated DNA fraction was collected and purified again in a second HPLC run to ensure maximum purification from unbound sulfo-SMCC.

densing the amine of the oligonucleotide with the NHS ester of sulfo-SMCC. In the second step, the maleimide is reacted with a thiol group on the nanocrystal surface to coval ently link the oligonucleotide to the particle surface.

Sulfo-SMCC was dissolved in dimethylformamide to approximately $70 \mathrm{mg} / \mathrm{mL}$ and added to amino-modified oligonucleotides (final DNA concentration about $50 \mu \mathrm{M}$, IDT, Coralville, IA) in 500-1500 molar excess (typically, 1000×) in $150 \mathrm{mM}$ sodium phosphate buffer, 100-200 mM (typically, $150 \mathrm{mM}$ ) $\mathrm{NaCl}, \mathrm{pH}$ 7.0. After incubating for $1 \mathrm{~h}$ at room temperature, bulk unreacted cross-linker molecules were removed by using a Sephadex G-25 column (NAP-25, Pharmacia Biotech, Piscataway, NJ ). Complete removal of excess cross-linker required two successive purification steps with anion-exchange chromatography (1100 Series HPLC system, Agilent, Roseville, CA) using a Zorbax column (Zorbax Bio Series Oligo, analytical column, Du Pont, Wilmington, DE). As shown in Figure 2, eluant fractions containing free crosslinker, unreacted DNA, and maleimide-activated DNA could be separated. The latter two fractions were unambiguously identified by matrix-assisted laser desorption ionization-time of flight (MALDI-TOF) spectroscopy. The eluant fraction containing the maleimide-activated DNA was collected, desalted using a Sephadex G-25 column, lyophilized, and stored at $-20^{\circ} \mathrm{C}$ under nitrogen. Reacting maleimide-activated DNA with thiol groups of mercaptopropionic acid and measuring the increase in mass by MALDI-TOF spectroscopy verified preserved activity of the maleimide.

Covalent coupling of maleimide-activated oligonucleotides to thiol-bearing nanocrystals was typically performed in 10$50 \mathrm{mM} \mathrm{NaCl}$ (typically, $25 \mathrm{mM}$ ) in 10-100 mM sodium phosphate buffer (typically, $25 \mathrm{mM}$ ), pH 7.3. The ratio of malei mide-bearing DNA to thiol-bearing nanocrystals ranged from 1:2 to 1000:1, as did the reaction time (several hours to 2 days; typically, 1 day) at room temperature.

Gel electrophoresis was used to verify coupling of oligonucleotides to nanocrystals. The oligonucleotide-derivatized nanocrystals were diluted in loading buffer containing $10 \%$ glycerol and were run in $0.5 \times$ tris-borate-EDTA buffer (no. a)

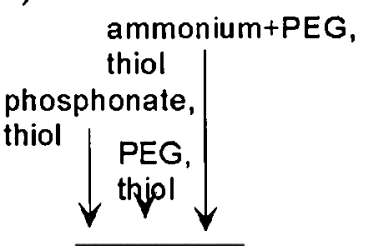

b)

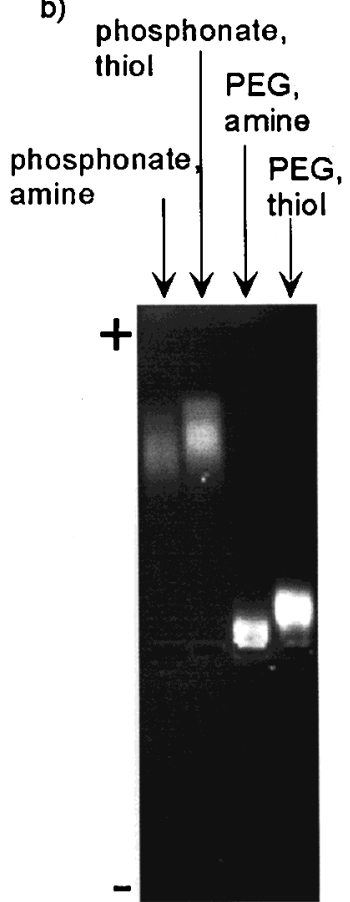

Figure 3. Gel electrophoresis of silanized nanocrystals. All nanocrystals shown in this figure originate from the same $\mathrm{CdSe} / \mathrm{ZnS}$ preparation. (a) Siloxane shells with different stabilization molecules. From left to right, bands of phosphonate silane, PEG-silane, and ammonium/PEG-silane-coated nanocrystals bearing surface thiols are shown. (b) Siloxane shells with different functional groups. Bands of phosphonate silane and PEG-silane-stabilized nanocrystals with either surface amines or thiols as functional groups are shown.

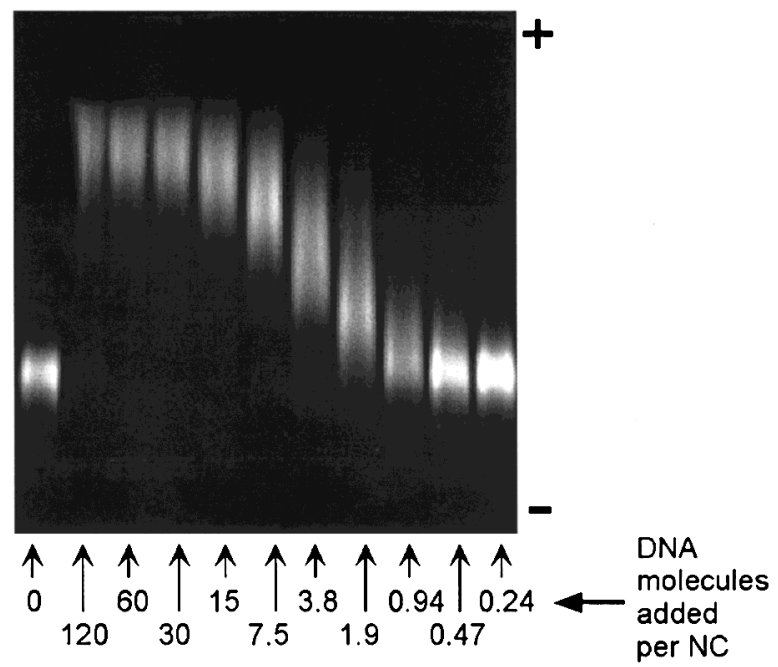

Figure 4. Verification of oligonucleotide conjugation by gel electrophoresis. PEG-silane-coated nanocrystals with yellow fluorescence incubated with different amounts of added malei mide-activated DNA (21 bases, single-stranded DNA, 1-day incubation time) were electrophoresed on a $1 \%$ agarose gel.

T3913, Sigma-Aldrich) on a $1 \%$ agarose gel at about $10 \mathrm{~V} / \mathrm{cm}$ for $1 \mathrm{~h}$. The gels were illuminated with an ultraviolet transilluminator and the fluorescence of the nanocrystals was imaged with a digital camera (Eagle Eye II System) (see Figures 4-8).

The conservation of the biological activity of oligonucleotides conjugated to nanocrystals was investigated with a hybridization experiment. ${ }^{22} \mathrm{~A}$ gold pattern was evaporated on a glass substrate. Thiol-modified oligonucleotides were attached to this 
ca. $2 \mathrm{~h}$ incubation time

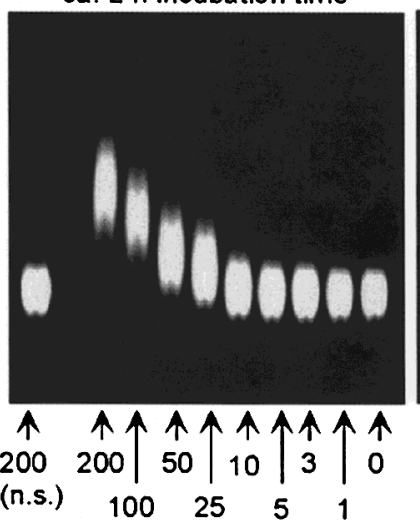

ca. $24 \mathrm{~h}$ incubation time

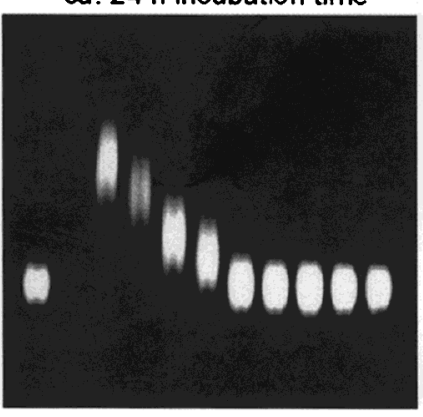

ca. $48 \mathrm{~h}$ incubation time

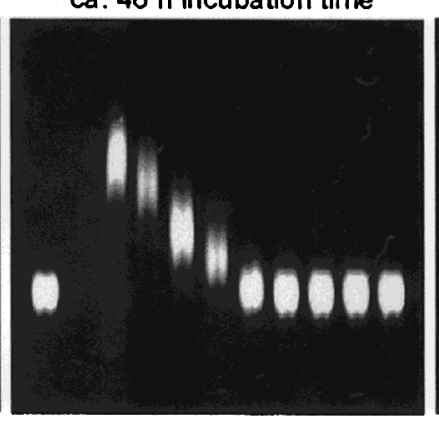

ca. $72 \mathrm{~h}$ incubation time

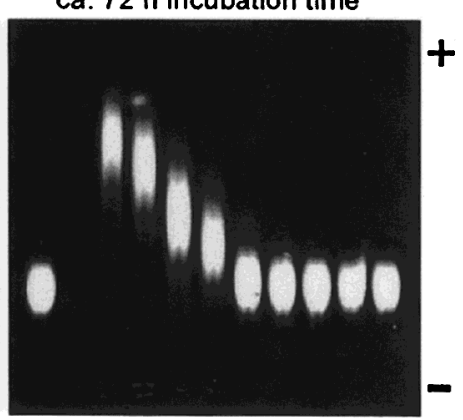

DNA molecules added per NC

Figure 5. Time dependence of the gel electrophoresis migration pattern. PEG-silane-coated nanocrystals incubated with different amounts of added maleimide-activated DNA (21 bases, single-stranded DNA) were electrophoresed on a 1\% agarose gel after incubation times of 2 h, 1 day, 2 days, and 3 days. In the left lane, unmodified DNA (i.e., without maleimide activation) was added to the nanocrystals to test for nonspecific binding.

pattern by thiol-gold bonds. Oligonucleotide-nanocrystal conjugates were added on top of the oligonucleotide-patterned substrate and incubated. After sufficient rinsing a fluorescence image of the surface of the substrate was recorded with an optical epifluorescence microscope. To test specific binding, oligonucleotides complementary to the oligonucleotides conjugated to the nanocrystals were used for patterning the substrate. To test nonspecific binding, oligonucleotides not complementary to the oligonucleotides conjugated to the nanocrystals were used for patterning the substrate.

\section{Results and Discussion}

Nanocrystals Synthesis. A tool kit of water-soluble nanocrystals with different charges and functional groups was developed through the modification of an existing protocol.$^{15} \mathrm{E}$ ither electrostatic or steric repulsion ensures the stability of the charged phosphonateand PEG/ammonium silane or the virtually uncharged PEG-silane-coated nanocrystals in aqueous solution, respectively. These concepts are well-known for the stabilization of latex particles and lipid vesicles. It is important to point out that the surface groups used for stabilization (phosphonate, PEG, ammonium) are independent of the groups used for functionalization $(-\mathrm{SH}$, $-\mathrm{NH}_{2},-\mathrm{COOH}$ ) (see Figure 1).

Agarose gel electrophoresis of the nanocrystals reveals the charge differences between the different types of surface derivatization because migration is strongly dependent on charge. Whereas negatively charged phosphonate silane-coated nanocrystals migrate toward the positive pole, positively charged PE G/ammonium silanecoated nanocrystals migrate toward the negative pole (Figure 3a). The virtually neutral PEG-silane-coated nanocrystals migrate slowly toward the positive electrode, which indicates a slight negative charge. This residual charge might be attributed to unquenched silanol $(-\mathrm{SiOH})$ groups in the siloxane shell, which are negatively charged at the $\mathrm{pH}$ of the gel $\mathrm{pH}$ 8-9. Mobility is reduced when amines are used as functional groups

(19) Zanchet, D.; Micheel, C. M.; Parak, W. J .; Gerion, D.; Alivisatos, A. P. Nanoletters 2001, 1, 32-35.

(20) Zhang, F.; Kang, E. T.; Neoh, K. G.; Wang, P.; Tan, K. L. J Biomed. Mater. Res. 2001, 56, 324-332.

(21) Melzak, K. A.; Sherwood, C. S.; Turner, R. F. B.; Haynes, C. A. J. Coll loid Interface Sci. 1996, 181, 635-644.

(22) Gerion, D.; Parak, W. J .; Zanchet, D.; Williams, S. C.; Micheel, C. M.; Alivisatos, A. P. J . Am. Chem. Soc., in press. rather than thiols (Figure $3 b$ ). This is likely to be caused by the positive charge of the amines at pH 8-9. PEGsilane and PEG/ammonium silane-coated nanocrystals show slightly brighter fluorescence than phosphonate silane-coated nanocrystals. This observation is in good agreement with the findings of other groups who have reported surface-charge effects on the quantum yield. ${ }^{11}$ PEG/ammonium silane-coated nanocrystals, however, are less stable in electrolyte solution than phosphonateand PEG-coated nanocrystals.

The ability to generate an array of nanocrystals with varied surface functionality offers many advantages over standard protocols, which typically produce only negatively charged nanocrystals with carboxyl groups. First, because gel electrophoresis is an established method used to verify the coupling of biological molecules to nanocrystal surfaces, ${ }^{19}$ it is beneficial to have nanocrystals with different intrinsic mobility. Thus, it is desirable to choose nanocrystals with an electrophoretic mobility that is sufficiently different from the mobility of the biomolecule itself, so that biomoleculenanocrystal conjugates can be easily separated by a mobility shift. Second, it is possi ble to optimize reaction conditions for the covalent attachment of biological macromolecules. To reduce nonspecific binding through electrostatic interactions, and thus enhance the probability of covalent bond formation, nanocrystals with a polarity equal to that of the biomolecules are desirable. For instance, a significant decrease in nonspecific adsorption can be expected during the conjugation of positively charged molecules to positively charged nanocrystals. On the other hand, when the biological molecules and nanocrystals bear opposite charges, electrostatic attraction promotes nonspecific particle adsorption. This has been applied for the adsorption of proteins modified with positively charged zippers to negatively charged nanocrystals ${ }^{10,11}$ and could now be extended to negatively charged zippers and positively charged nanocrystals. Third, nonspecific binding to cells depends on the charge of the siloxane shell, ${ }^{6}$ and can be controlled by mani pulating the surface charge of the nanocrystals. Because of the similar surface structure between PEG and water, PEG-coated nanocrystals are promising candidates for fluorescent cell labeling with reduced nonspecific adsorption. ${ }^{20}$ 

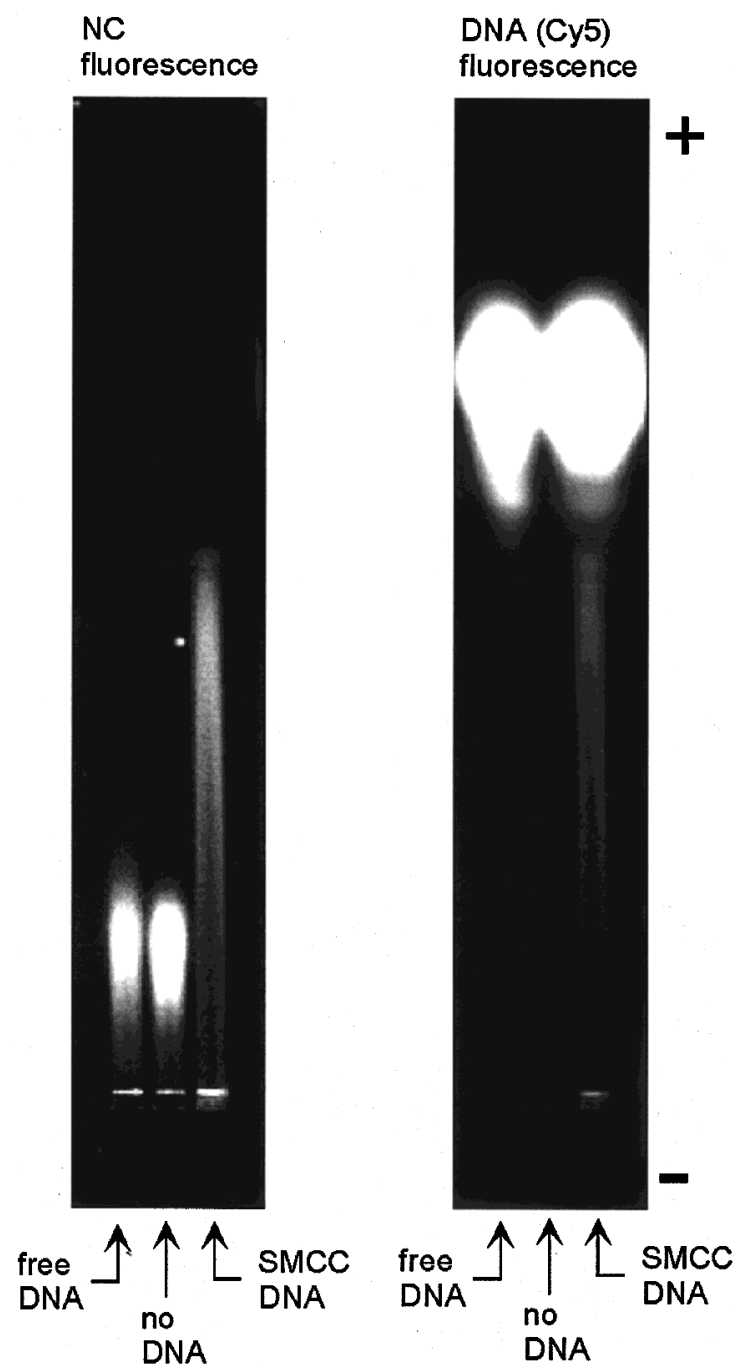

Figure 6. Colocalization of DNA and nanocrystals in the gel electrophoresis migration pattern. PEG-silane-coated nanocrystals were incubated with maleimide-activated and unmodified DNA (30 bases, single-stranded DNA with Cy5 label, added DNA/nanocrystals ratio $=500$, 1-day incubation time) and electrophoresed on a $1 \%$ agarose gel. By using two sets of filters, the location of nanocrystals (green excitation, yellow fluorescence, left panel) and the DNA (red excitation, red fluorescence originating from the Cy5-molecules bound to the DNA, right panel) in the migration pattern could be determined separately. The three lanes in the gel correspond to nanocrystals incubated with unmodified DNA, just nanocrystals, and nanocrystals incubated with maleimide-activated DNA. No unmodified DNA is attached to the nanocrystals (right panel, left lane) and the nanocrystals migrate similar to the nanocrystals without added DNA (left panel, left and middle lanes). Nanocrystals incubated with maleimideactivated DNA migrate faster than control nanocrystals (left panel, middle and right lanes). DNA and nanocrystals are colocalized (left and right panels, right lane), which indicates that DNA is specifically bound to the nanocrystals. The overall binding efficiency is low, because most of the maleimideactivated DNA does not bind to the nanocrystals but migrates similarly to free DNA (right panel, left and right lanes). Free DNA migrates faster than DNA-nanocrystal conjugates. To visualize the DNA attached to the nanocrystals, the image of the gel has been overexposed.

Bioconjugation. Coupling of oligonucleotides in several concentrations to PEG-silane-coated nanocrystals is shown in Figure 4. Similar results were obtained for four nanocrytal samples of different emission color
NC

fluorescence
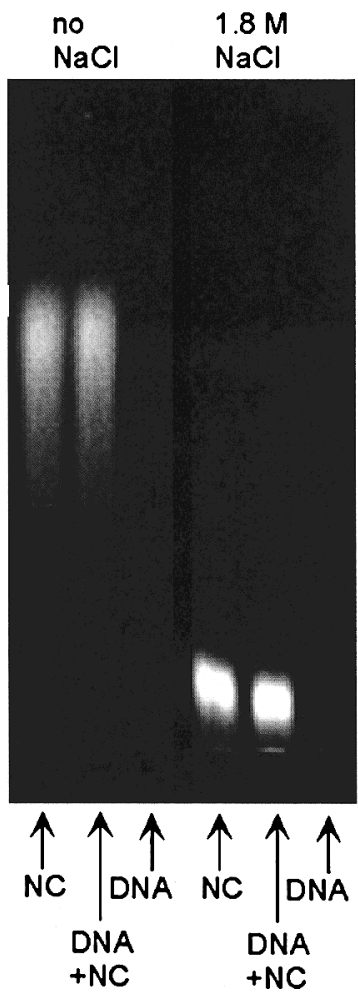

DNA (Cy5)

fluorescence

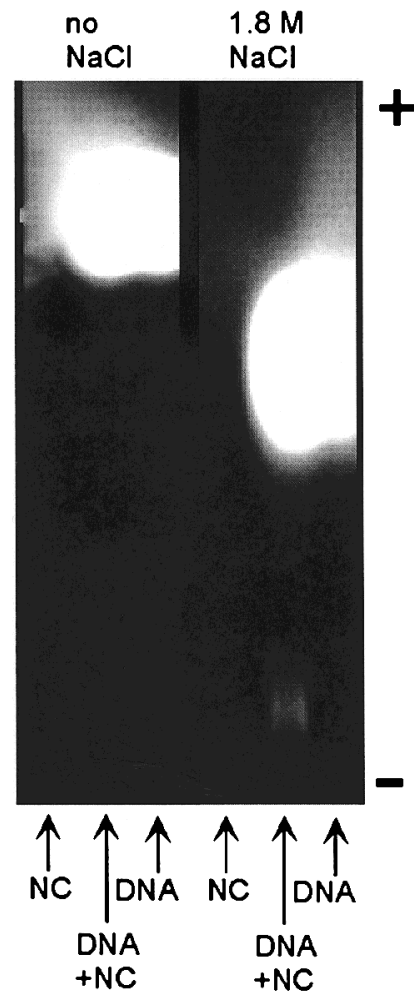

Figure 7. Nonspecific DNA adsorption to nanocrystals. Phosphonate silane-coated nanocrystals were incubated with unmodified DNA (20 bases, double-stranded DNA with Cy5 label, 1-day incubation time) either with 0 or $1.8 \mathrm{M} \mathrm{NaCl}$ concentration and electrophoresed on a $1 \%$ agarose gel. By using two sets of filters, the location of nanocrystals (green excitation, yellow fluorescence, left panel) and the DNA (red excitation, red Cy5-fluorescence, right panel) in the migration pattern could be determined separately. The left and right three lanes in the gel correspond to just nanocrystals, nanocrystals with unmodified DNA, and just free DNA, incubated in $\mathrm{NaCl}$ concentrations of 0 and $1.8 \mathrm{M}$, respectively. For high salt concentrations the band of the nanocrystals is retarded compared with the band of the nanocrystals incubated without $\mathrm{NaCl}$ (left panel). This can be explained by partial agglomeration of the nanocrystals. ${ }^{15}$ No unmodified DNA is attached to the nanocrystals (right panel), and the nanocrystals migrate similar to the nanocrystals without added DNA (left panel) for $\mathrm{c}(\mathrm{NaCl})=0$. For very high salt concentrations $[\mathrm{c}(\mathrm{NaCl})=$ 1.8 M] some DNA is colocalized with nanocrystals (left and right panels, middle of the three right lanes), which indicates that DNA is bound to the nanocrystals. Because the DNA was unmodified, i.e., without any specific binding site, this binding has to be considered as nonspecific. To visualize the DNA attached to the nanocrystals, the image of the gel has been overexposed.

and for four oligonucleotide sequences with 20-100 bases length. Underivatized PEG-coated nanocrystals are almost neutral in charge and thus only marginally migrate through the gel. Derivatization with negatively charged oligonucleotides, however, results in substantially increased migration depending on the amount of oligonucleotide coupled to the nanocrystal. Saturation in gel mobility was not observed in some nanocrystal batches with DNA:nanocrystal ratios of up to 200 . Migration patterns did not modify with prolonged incubation; after a few hours incubation the gel mobility was found to be independent of the incubation time, see Figure 5. The attachment of DNA to nanocrystals 


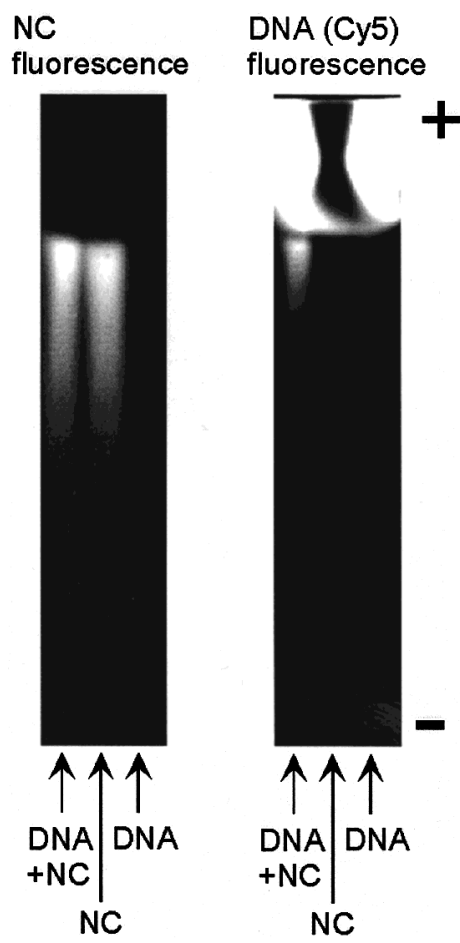

Figure 8. Oligonucleotide conjugation of negatively charged nanocrystals. Phosphonate silane-coated nanocrystals were incubated with malei mide-activated and unmodified DNA (30 bases, double-stranded DNA with Cy5 label, 1-day incubation time) and electrophoresed on a $1 \%$ agarose gel. By using two sets of filters, the location of nanocrystals (green excitation, yellow fluorescence, left panel) and the DNA (red excitation, red Cy5-fluorescence, right panel) in the migration pattern could be determined separately. The three lanes in the gel correspond to nanocrystals incubated with maleimide-activated DNA, just nanocrystals, and just maleimide-activated DNA. DNA and nanocrystals are colocalized (left and right panels, left lane), which indicates that maleimide-activated DNA is specifically bound to the nanocrystals. The overall binding efficiency is low, because most of the maleimide-activated DNA does not bind to the nanocrystals but migrates similar as free DNA (right panel, left and right lanes). Free DNA migrates only slightly faster than DNA-nanocrystal conjugates and negatively charged nanocrystals al one, which encumbers the separation of plain and DNA-conjugated nanocrystals. To visualize the DNA attached to the nanocrystals, the image of the gel has been overexposed.

therefore seems to be stable with time, which is to be expected from a covalent bond.

A series of control experiments was performed to further verify the specificity of the cross-linking reac- tion. For native DNA lacking maleimide activation, there was no shift in the nanocrystal band (Figures 5 and 6). Interestingly, at higher salt concentrations $(\gg 200 \mathrm{mM}$ ), nonspecific DNA adsorption was observed, even for negatively charged nanocrystals (Figure 7). ${ }^{21}$ Similar results were obtained for several DNA sequences and for double-stranded DNA. By using oligonucleotides labeled with an organic dye, it was shown that unbound DNA migrated much faster than the nanocrystal-DNA conjugates and that DNA and nanocrystals were indeed colocalized in the bands ascribed to the conjugates (Figure 6). All maleimide-activated DNA was double-high-performance liquid chromatography (HPLC)-purified to ensure the complete removal of unreacted cross-linker (Figure 2). The DNA-nanocrystal conjugates were stable in el ectrolytic solution for at least one month, and the fluorescence of the nanocrystals was minimally affected by the conjugation. These results demonstrate that DNA can be specifically and covalently bound to water-soluble nanocrystals.

In the conjugation of DNA to Au particles, we have been able to count the number of DNA strands bound per Au particle, and to isolate conjugates with discrete numbers. ${ }^{19}$ So far we have not been able to do this with the silanized semi conductor nanocrystals, but work on this is ongoing. However, when the entire nanocrystal band is shifted for high concentrations of added DNA, there must be at least one DNA molecule attached to each nanocrystal. Preliminary results involving oligonudeotides that are labeled with organic dyes suggest a low overall binding efficiency, with only a few DNA strands bound per nanocrystal on average (see Figure 6).

The gel electrophoresis experiments described above rely on the near-neutral nature of the PEG coating to allow electrophoretic mobility to be driven by the presence of negatively charged DNA on the particle surface. Similar electrophoresis experiments with DNAderivatized, phosphonate-coated nanocrystals demonstrated negligible decrease in mobility after DNA conjugation (Figure 8). This is attributed to the intrinsic mobility of the negatively charged phosphonate-coated nanocrystals, which migrate with nearly the same mobility as unconjugated DNA. Because both DNA and underivatized nanocrystals have similar electrophoretic mobility, and the nanocrystal bands are smeared and diffuse, which masks subtle shifts in migration due to conjugation, the coupling of DNA to these nanocrystals could not be verified by gel electrophoresis. The elec- a)

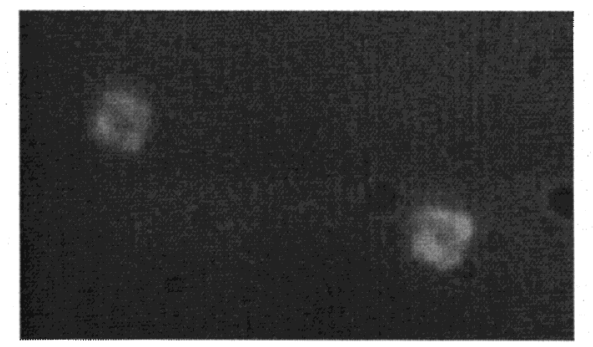

b)

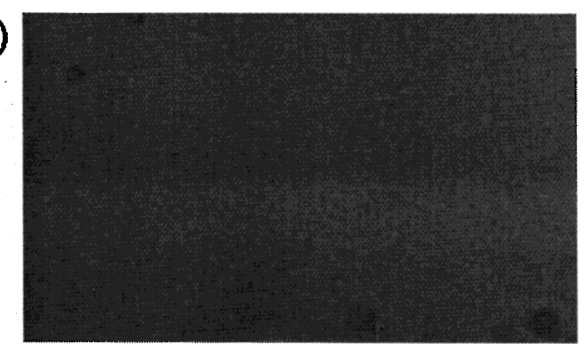

Figure 9. Hybridization of DNA-nanocrystal conjugates with surface-bound single-stranded DNA. Thiol-modified DNA (21 bases, single-stranded DNA) was attached to a gold-patterned glass substrate (hol low squares of $8 \times 8 \mu \mathrm{m}$ ). DNA-nanocrystal conjugates (single-stranded DNA, 21 bases, green fluorescent nanocrystals) were added on top of the substrate and incubated. After a rinsing step, the fluorescence of the substrate was recorded with an optical microscope. (a) The oligonucleotides bound to the surface of the substrate are complementary to the oligonucleotides conjugated to the nanocrystals. (b) The oligonucleotides bound to the surface of the substrate are not complementary to the oligonucleotides conjugated to the nanocrystals. 
trophoretic mobility of the PEG-coated, DNA-derivatized nanocrystals seems different than the electrophoretic mobility of DNA gold-nanocrystal conjugates. ${ }^{19}$ Because the water-soluble gold nanocrystals are coated with negatively charged phosphine, they migrate nearly as far as unconjugated DNA. The addition of further negative charge to these already highly charged particles after ol igonucleotide conjugation did not enhance their electrophoretic mobility. Rather, migration through the gel was reduced because of an increase in particle diameter. This is in contrast to the PEG-coated semiconductor nanocrystals described in this study, where oligonucleotide coupling increases mobility because of significantly increased negative charge.

Oligonucleotidenanocrystal conjugates were shown to bind to complementary oligonucl eotides immobilized on a surface, but not to noncomplementary oligonucleotides (see Figure 9). This demonstrates that oligonucleotides conjugated to nanocrystals retain their biological activity, i.e., their ability to form Watson-Crick base pairs is still functional. Furthermore the binding is specific, because only complementary sequences bind. A more detailed investigation is reported el sewhere. ${ }^{22}$

Single- and double-stranded DNA were covalently bound to the surface of PEG-coated semiconductor nanocrystals, and coupling was verified by using gel electrophoresis. Although derivatization of DNA to nanocrystals has been demonstrated by others, ${ }^{7,8}$ the protocol described here has a conceptual advantage. Mitchell et al. reported direct conjugation of oligonucleotides to the surface of the CdSe/ZnS nanocrystals by using thiol groups. Thiol-ZnS bonds are not as stable as thiol-gold bonds, thus DNA is bound and released in a dynamic process. Pathak et al. describe a more advanced procedure, in which oligonucleotides were covalently attached to the outer layer of water-soluble nanocrystals, which were first stabilized with a layer of dithiothreitol. Although the DNA was thus bound to the ZnS surface with two instead of just one thiol-ZnS bond, similar to an idea reported by the Bawendi group, ${ }^{10}$ these nanocrystals are likely to be less stable in electrolytic solution than nanocrystals that are stabilized by multiple thiol-ZnS bonds and a crosslinked siloxane shell. The principle advantage of the silanized nanocrystals described in this study is the exceptional stability of the bioconjugates. These nanocrystals are stable in electrolytic solution and do not preci pitate or agglomerate for months, their photophysical properties are conserved, and oligonucleotides can be covalently bound to the nanocrystal surface rather than passively adsorbed. Preliminary results show that oligonucleotide-decorated nanocrystals can be hybridized to surface-bound complementary ol igonucleotides, paving the way for the use of DNA-nanocrystal conjugates as fluorescence probes for specific multicolor labeling.

\section{Conclusions}

In this study, a flexible synthesis of versatile watersoluble nanocrystals with siloxane shells of different properties has been described. Virtually any surface charge can be achieved by combining different ratios of neutral or negatively or positively charged siloxanes in the outer coat of the nanocrystals. In addition, a variety of functional groups for conjugation can be incorporated into the nanocrystal surface and the nanocrystals can be used to fluorescently label virtually any biological molecule. A distinguishing feature of this methodology is that the nanocrystals used for creating nanocrystalbiomolecule conjugates-identical except for the surface charge or functionalization-can be prepared by adjusting one protocol through choice of different silane compositions. Through conjugation of appropriate receptors or ligands, nanocrystals could be targeted to specific molecules, organelles, or tissues. Nanocrystal-labeled oligonucleotides could be used to probe complementary oligonucleotides in DNA arrays, sectioned tissues, or nitrocellulose blots. The ability to conjugate any of an array of biological molecules would allow multicolor staining of cells and intracellular compartments in a single staining procedure, and provide expansive staining and imaging applications in biology.

Acknowledgment. W. J . P. acknowledges the German Research Foundation (DFG) for a postdoctoral fellowship. D. Z. is grateful to FAPESP, proc. 99/086037, for financial support. T. P. is supported by the Seconda Universita Degli Studi Di Napoli (Caserta, Italy). C. M. M. is a Howard Hughes Medical Institute Predoctoral Fellow. S. C. W. acknowledges the Lawrence Livermore National Laboratory and the National 7Physical Science Consortium. This work was supported by NIH National Center for Research Resources, Grant 1 R01 RR-14891-01 through the U.S. Department of Energy under Contract DE-AC03-76SF00098, DOD Advanced Research Projects Agency (DARPA) under Grant N00014-99-1-0728, and by the Director, Office of Energy Research, Office of Science, Division of Materials Sciences, of the U.S. Department of Energy under Contract DE-AC03-76SF 00098.

CM 0107878 